\title{
Pushing, pulling, and squeezing our way to understanding mechanotransduction
}

\author{
Michael J. Siedlik ${ }^{1}$, Victor D. Varner ${ }^{1}$, and Celeste M. Nelson ${ }^{1,2^{*}}$ \\ Departments of ${ }^{1}$ Chemical \& Biological Engineering and ${ }^{2}$ Molecular Biology \\ Princeton University, Princeton, NJ 08544
}

(*) Address correspondence to C.M.N.

303 Hoyt Laboratory, William Street

Princeton, NJ 08544

Tel: 609-258-8851

Fax: 609-258-1247

E-mail: celesten@princeton.edu

Keywords: mechanical stress; morphogenesis

\begin{abstract}
Abbreviations: 2D, two-dimensional; 3D, three-dimensional; Cry2, cryptochrome 2; ECM, extracellular matrix; FAK, focal adhesion kinase; FRET, fluorescence resonance energy transfer; MRTF, myocardin-related transcription factor; SRF, serum response factor; TAZ, transcriptional coactivator with PDZ-binding motif; TFM, traction force microscopy; YAP, yes-associated protein
\end{abstract}




\begin{abstract}
Mechanotransduction is often described in the context of force-induced changes in molecular conformation, but molecular-scale mechanical stimuli arise in vivo in the context of complex, multicellular tissue structures. For this reason, we highlight and review experimental methods for investigating mechanotransduction across multiple length scales. We begin by discussing techniques that probe the response of individual molecules to applied force. We then move up in length scale to highlight techniques aimed at uncovering how cells transduce mechanical stimuli into biochemical activity. Finally, we discuss approaches for determining how these stimuli arise in multicellular structures. We expect that future work will combine techniques across these length scales to provide a more comprehensive understanding of mechanotransduction.
\end{abstract}




\section{Introduction}

Biological information is relayed across multiple length scales_-between nearby subcellular compartments, within individual cells, and across multicellular structures—via chemical, electrical, and mechanical signals. These signals, which include diffusible molecules, gradients in electrical potential, and physical forces that depend upon local material properties, are highly integrated and are often transduced into different forms. The conversion of a mechanical stimulus into a biochemical response can regulate gene expression and enzyme activity [1-3]. It is well appreciated that this transduction of mechanical signals, known as mechanotransduction, is crucial in many biological contexts, including development $[4,5]$, disease progression [6, 7], wound healing [8], and tissue homeostasis [9].

In their native physiological setting, cells reside within much larger structures and are subject to mechanical stimuli generated by and transmitted through the corresponding tissue and microenvironment. Key nodes of mechanotransduction within cells include sites of cell adhesion, tension-dependent transcription factors, and stretch-activated receptors and ion channels that are located in the plasma membrane (Fig. 1). At focal adhesions, the activation of proteins such as focal adhesion kinase (FAK) depends upon the mechanical context. FAK is phosphorylated in cells under tension and binds various target molecules, including other focal adhesion proteins, to initiate downstream signaling [10]. Elsewhere, yes-associated protein (YAP) and transcriptional coactivator with PDZ-binding motif (TAZ), are dephosphorylated in response to increased substratum rigidity and preferentially localize to the nucleus [11, 12]. Similarly, the activity of myocardin-related transcription factor (MRTF)-A, a cofactor of serum response factor (SRF), is regulated by the concentration of monomeric actin, thereby enabling it to influence 
transcription in response to stress-mediated increases in actin polymerization $[13,14]$. Finally, mechanosensitive ion channels, such as Piezo-1 and Piezo-2, are responsible for sensing shear stress in vascular endothelial cells [15], sensing touch in peripheral neurons [16], and sensing nanotopography in hippocampal neurons [17]. In general, the mechanisms by which such modules respond to mechanical cues and function in the context of processes that span larger length and time scales remain enigmatic.

Mechanical forces act across multiple length scales. For this reason, experimental approaches are required to measure mechanotransduction from the molecular level to the tissue level. This review highlights techniques from biophysics, mechanobiology, and biomechanics that have clarified and quantified mechanotransduction across different length scales. Specific examples of mechanotransductive pathways are not discussed in detail here. For this, interested

readers are directed to a number of recent reviews $[4,9,18,19]$. Rather, we highlight experimental techniques that actively probe mechanically-dependent molecular and cellular activity, as well as techniques that passively measure forces, force-dependent changes in molecular orientation, and the resulting effect on downstream signaling. The reader will be directed to more in-depth reviews focusing on the relevant biology or experimental techniques where possible. Starting from methods aimed at probing mechanotransduction on the molecular scale, we gradually move up in length scale to discussion of cell- and tissue-level techniques.

\section{Molecular-scale methods to investigate mechanotransduction}

It is generally thought that mechanical forces induce changes in protein conformation, thus revealing hidden (or cryptic) binding sites or removing autoinhibitory domains to enable 
mechanically-sensitive protein interactions and functions [20,21]. Such changes in protein conformation occur in both intracellular and extracellular contexts. As demonstrated in vitro, force applied across the intracellular protein talin can unfold its rod domain, revealing cryptic binding sites for vinculin [22]. Conversely, force transmitted across focal adhesions can induce changes in the conformations of extracellular proteins, as exemplified by fibronectin fibrillogenesis [23, 24]. In either case, uncovering specific molecular events requires the measurement and manipulation of force as well as the ability to detect and apply molecular-scale conformational changes.

Given that applying loads to individual molecules drives changes in their conformations, many techniques have been developed to clarify and define these mechanotransducing events in highly controlled, yet often simplified, situations [21]. To manipulate protein conformation, such setups often involve optical or magnetic tweezers [25-27]. Optical tweezers, or optical trapping, relies on orienting the radiation pressure of a focused laser beam incident on a microsphere (Fig. 2A). When properly calibrated and operated in the linear force-response regime, the force applied to the bead by the optical trap is proportional to the displacement of the bead from the center of the trap. By trapping particles tethered to a protein of interest, one can move the particle in three dimensions (relative to a reference point) to apply force across the protein and thus observe force-dependent changes in protein activity.

The use of optical traps in the investigation of mechanotransduction is highlighted by a recent study of the tension-dependent lifetime of bonds between the cadherin-catenin complex and filamentous actin [28]. This experimental setup consisted of attaching fluorescently-labeled 
E-cadherin, $\beta$-catenin, and $\alpha$ E-catenin to glass microspheres mounted on a coverslip. A single phalloidin-coated actin filament was held in place between two additional beads immobilized in optical traps. Tension was applied to bonds between the cadherin-catenin complex and actin by moving the coverslip parallel to the actin filament. Bond forces were read out from the displacement of the beads in the optical traps and related to bond lifetimes, which were determined from temporal data of the force exerted on the trapped beads. This study demonstrated that bonds between the cadherin-catenin complex and filamentous actin are initially weak and quickly dissociate, but rapidly transition to a strongly bound state under increasing loads, a defining feature of catch bonds [29]. In this way, experiments using optical tweezers enable one to manipulate protein interactions and activity by applying preciselymeasured forces.

Similar to optical trapping, magnetic tweezers involve controlling a magnetic particle via an applied magnetic field [26, 27, 30, 31]. For a superparamagnetic particle, the force applied by the magnetic trap is proportional to the gradient of the magnetic field (Fig. 2B). Magnetic trapping as a tool for uncovering force-dependent protein binding is highlighted by a recent study that investigated the binding of vinculin to talin [22]. Here, talin was attached between a glass surface and a magnetic bead. The suspended talin was exposed to the fluorescently-labeled binding domain of vinculin and force was applied across the protein by magnetically manipulating the bead. Binding of vinculin was determined by fluorescence photobleaching, during which it was observed that greater photobleaching occurs in the presence of force. Thus, this study demonstrated that application of physiologically relevant forces across talin rods 
exposed cryptic binding sites for vinculin. The high degree of control provided by optical and magnetic trapping has made these techniques valuable for studies in vitro.

Complementary to the in vitro application of force across individual molecules, conformational changes and molecular scale forces have been monitored in living cells. Techniques that take advantage of fluorescence resonance energy transfer (FRET) yield information about the conformation of proteins by quantifying the relative transfer of energy between nearby fluorophores within or near the protein of interest (Fig. 2C) [32, 33]. Recent reviews [34] discuss FRET technology in-depth and so the following discussion will highlight tools that detect forces, conformational changes, and tension-dependent changes in protein activity.

One of the most common techniques for measuring force across a protein is by engineering a variant of that protein to contain a FRET-based tension-sensing module (see [34] for more information). Such modules typically consist of a donor fluorophore separated from an acceptor fluorophore by an elastic linker domain. The sequence of this domain determines the mechanical properties of the tension sensor and thus specifies its sensitivity to force. Forces are calculated by first quantifying the relative intensities of the two fluorophores or the fluorescence lifetime of the donor with and without the acceptor. This information can be related to the FRET efficiency [35], defined as the proportion of excitation energy transferred from the donor to the acceptor fluorophore. FRET occurs non-radiatively and this efficiency corresponds to the distance between fluorophores. For a properly calibrated tension sensor, the measured FRET 
efficiency can then be related to the force acting across the tension-sensing module and is indicative of the tension present across the protein.

A recent study demonstrating the power of FRET-based techniques incorporated a tension-sensing module into vinculin (Fig. 2C) [36]. Here, the tension-sensing module consisted of a cyan fluorescent protein variant as the donor fluorophore and a yellow fluorescent protein variant as the acceptor fluorophore, separated by a flagelliform linker domain. The resulting vinculin tension sensor could be used to measure pico-Newton $(\mathrm{pN})$ levels of force across this protein in stable focal adhesions. This construct was subsequently incorporated into vinculindeficient fibroblasts and endothelial cells, in which focal adhesion assembly and disassembly could be observed while tension was monitored across vinculin during the dynamic process of cell migration. These methods enabled the measurement of intramolecular force in relation to changes in protein binding and revealed that higher levels of tension across vinculin correlate with focal adhesion assembly whereas lower levels occur during disassembly. Similar tensionsensing modules have been used with E-cadherin [37] and $\beta$-spectrin [38] and so it seems likely that these FRET-based tools will continue to provide molecular-scale insight into forcedependent protein interactions.

In addition to quantifying intramolecular tension, FRET-based sensors can also relate force-induced changes in molecular conformation to protein binding. Such sensors have been used to investigate mechanotransduction involving $\alpha$-catenin [39], vinculin [40], and fibronectin [23, 41]. As opposed to the aforementioned tension sensors which use fluorophores separated by a linker domain, constructs in these studies need not be designed with a separate linker. Changes 
in protein conformation are typically qualitative, with decreased FRET between fluorophores incorporated within or tethered to a protein usually sufficient to indicate an open conformation, whereas increased FRET typically coincides with a closed conformation. Such a construct has been used to investigate the response of fibronectin to cell-generated forces (Fig. 2C) [23, 24, 42]. Here, purified fibronectin was labeled with donor and acceptor fluorophores at specific amino acid residues. During cell culture, FRET measurements indicated whether the labeled fibronectin, which was coated on a coverslip, existed in a compact or elongated conformation. These methods were used to demonstrate that fibronectin stretches and unfolds in response to cellular forces, thus exposing new binding sites. Given that such sensors can arise from either engineered protein variants or by labeling unmodified molecules, FRET-based sensors provide a robust and versatile means to detect force-dependent changes in protein conformation.

Though FRET-based techniques offer quantitative insight into the molecular-scale events that drive mechanotransduction, certain challenges and obstacles must be considered. The sensors must be designed such that endogenous protein function is not compromised.

Additionally, deformations within the tension probes are assumed to be purely one-dimensional [43], though changes in fluorophore orientation also affect FRET [34]. Interestingly, this principle has been used in FRET tension sensors that evaluate forces based upon FRET readouts indicative of fluorophore rotation $[44,45]$. Demonstrated in actin and spectrin, these FRET constructs often contain an inelastic linker domain so as to mitigate confounding effects arising from changes in the distance between fluorophores. Nevertheless, FRET-based tools remain an exciting and promising means to elucidate molecular scale mechanotransduction in physiologically-relevant intracellular, and potentially in vivo, microenvironments. 


\section{Cellular-scale tools to probe mechanotransduction}

On the molecular scale, force-dependent changes in molecular conformation influence protein binding or activity. These mechanosensitive molecular events propagate to the scale of the cell to influence cell behavior, including migration, differentiation, and survival.

Understanding mechanotransduction at this level thus requires quantifying mechanical forces applied to or exerted by regions of the cell while monitoring intracellular responses to such stimuli. Here, we highlight techniques that measure cell-generated forces, quantify changes in protein localization or function in response to force, and generate mechanical loads within the cell.

Measuring cell-generated forces often involves measuring deformations or displacements of a reference object. For example, traction force microscopy (TFM) has been used to resolve forces exerted by single cells on the surrounding microenvironment in two [46] or three [47, 48] dimensions by monitoring displacements of fluorescent markers embedded within the substratum or deflections of flexible microscale posts (Fig. 3A). If the material properties of the substratum are known, stresses can be calculated from the displacement fields [46, 49-52]. A recent example highlighting this technique quantified cardiomyocyte contractions using an array of elastomeric microposts $[53,54]$. The tips of these microposts were coated with extracellular matrix (ECM) protein while the sides were fluorescently labeled to enable visualization of post displacements. By recording high-speed video of the contracting cells, the authors could track micrometer-scale displacements with temporal resolution of tens of milliseconds. This study demonstrated simultaneous quantification of contractile force, velocity, and power produced by 
cardiomyocytes at each of their integrin-based cell-matrix adhesion points. Similar techniques, such as surface-adhered DNA- [55], polypeptide- [56], and polyethylene glycol-based probes [57] can map traction forces at even higher resolution —in this case, pN-level forces can be measured across individual focal adhesions and membrane receptors.

One promising new technique for measuring forces on the level of individual cells involves injecting deformable fluorescent oil microdroplets into multicellular aggregates (Fig. 3B) [58]. These oil droplets were formed from biocompatible fluorocarbon oil in the presence of biotinylated surfactant and filtered to produce precursor droplets with radii on the order of ten micrometers. The exposed biotinylated surface of each droplet was then linked to biotinylated RGD peptides or E-cadherin antibodies via fluorescent streptavidin, which has a high affinity for biotin. Droplet curvatures were determined from 3D reconstructions of the streptavidin fluorescence readout and the interfacial tension was determined prior to experiments, thereby enabling cell-generated forces to be calculated from a force balance using Laplace's law. This technique was used to measure stresses generated by individual cells within embryonic tissue, thereby demonstrating the ability to measure forces in vivo, an advantage over traditional TFM. Nevertheless, both techniques have advantages that make them well-suited for measuring cellgenerated stresses. TFM provides highly-controlled conditions that facilitate high-resolution measurements of stresses in a manner compatible with traditional fluorescence imaging methods. The fluorescent oil droplets, described above, are applicable for measuring 3D stresses in cell culture, embryonic tissues, or adult organs. 
Besides measuring forces, sensing protein activation in response to mechanical stimuli is also important for understanding mechanotransduction on the level of a cell. FRET-based sensors, in addition to detecting conformational changes in response to forces across a specific protein, have also been used to detect activation of mechanosensitive proteins at the cellular level. For example, to detect activation of FAK, a FRET-based biosensor was created that consists of a FAK-binding region flanked by donor and acceptor fluorophores [59, 60]. FAKdependent conformational changes of the biosensor influence the FRET readout, thereby enabling quantification of mechanosensitive FAK activity in live cells. The use of this construct has revealed that tension-dependent FAK activation is upstream of growth factor-induced FAK activity and sensitive to the type of ECM protein in contact with cell adhesions. FRET-based activity sensors have been applied to other mechanosensitive situations, including the activation of small GTPases involved in cell motility [61], the cytoplasmic activation of Src in response to increased focal adhesion tension [62], and histone methylation experienced by tumorigenic cells on a stiff substratum [63]. Taken together, FRET technology has thus provided valuable insight on the level of mechanosensitive protein interactions as well as on the level of cellular responses to internal and external mechanical stress.

The techniques described so far have been used to measure force or force-induced changes in protein activity on the scale of individual cells. Another meaningful experimental strategy will be to manipulate cellular forces and observe how these perturbations propagate into mechanosensitive signaling and changes in cell behavior. Because cells respond to the mechanical properties of their underlying substratum by adjusting cytoskeletal and junctional organization [64], one approach to probe intracellular mechanosensitive responses is to alter 
these properties. Whereas many studies have cultured cells on irreversibly cross-linked hydrogels [65], one recent study used photo-tunable hydrogels to provide insight into how cells respond to environments with dynamically changing mechanical properties (Fig. 3C) [66]. Here, the authors used alginate, which reversibly crosslinks in the presence of divalent cations. A solution of highly-concentrated calcium and gold nanorods were encapsulated in temperature-sensitive liposomes that could be ruptured by near-infrared light-mediated heating of the nanorods. Thus, light exposure could release the calcium, trigger alginate cross-linking, and increase the stiffness of the gel. This process could be reversed by incorporating a calcium chelator solution in the liposomes, in which case light exposure triggered calcium sequestration and gel softening. The first system to model dynamic changes in ECM stiffness, this study demonstrated temporal control over the morphology of fibroblasts cultured on or within these gels. The implantable nature of this system makes it compatible with studies in vivo, suggesting that it could be used to probe cellular mechanotransduction during processes such as development and disease progression.

Microfluidic pipette aspiration is another technique by which force can be directly applied to a cell so as to observe changes in mechanosensitive behavior. Classically used as a means to evaluate the bulk mechanical properties of cells, this method is based upon applying precise suction pressure to cells that are adhered to beads or in solution (Fig. 3D) [67]. Depending on the viscoelasticity of the cell, the effective viscosity or modulus can be determined from the suction pressure, the membrane curvature inside and outside of the micropipette, and the displacement of the membrane within the pipette. Recently, this technique has been expanded to studies of cell-level mechanotransduction in the investigation of stretch-activated ion channels 
[68], protein localization in response to force applied to the nucleus [69], and cadherin binding affinities between cells [70]. The first example [68] highlights the use of micropipette aspiration in conjunction with a novel microfluidic platform to facilitate high-throughput mechanical analysis of cells. In this study, cells were perfused through the microfluidic device and trapped for aspiration at built-in micropipette channels. Though the material properties of the cells were determined as described above, this study was also able to quantify the threshold levels of tension required to activate the stretch-activated ion channel MscL. This was determined by the uptake of a membrane-impermeable dye, propidium iodide, which fluoresced when bound to nucleic acid. By imaging this fluorescence as a function of the applied suction pressure, the threshold membrane tension required to activate this ion channel was estimated to be on the order of $\mathrm{mN} \mathrm{m}^{-1}$. Given their compatibility with other imaging techniques, methods that directly manipulate or apply forces to cells, such as modified substrata, micropipette aspiration, and atomic force microscopy $[71,72]$, can thus be used to elucidate cellular responses to mechanical stimuli.

Though not used in any mechanotransduction studies to date, the emerging field of optogenetics has enticing potential for perturbing intracellular forces. For example, a recentlydeveloped optogenetic probe of RhoA-mediated contractility in mammalian cells [73] may enable the observation of cellular effects that result from force-generating signaling networks (Fig. 3E). This construct consists of a photoresponsive Cryptochrome 2 (Cry2) protein fused to the small GTPase RhoA, which acts through its downstream effectors to induce the activation of myosin II and the associated cellular contractile machinery [74]. Here, Cry2 homo-oligomers form in response to illumination at 450 or $488 \mathrm{~nm}$, facilitating the oligomerization and 
subsequent activation of RhoA. Furthermore, these tools are continually evolving, as engineered variants of the Cry2 system have yielded longer-lived oligomerization [75]. Other potentiallyrelevant optogenetic tools include a photoactivatable form of Rac1 [75], which induces actin polymerization through the downstream effectors of that GTPase, and light-gated ion channels $[76,77]$, which could potentially trigger calcium-mediated contractile forces. Regardless of the construct to be used, certain challenges with light delivery remain to be addressed. Specifically, while delivering the photo stimulus to a cell in $2 \mathrm{D}$ culture is well established, delivering light to specific cells in a 3D culture or tissue remains nontrivial. If delivery issues can be balanced with maintaining low phototoxicity, optogenetic tools might provide key insight into cell-scale mechanotransduction in more physiologically-relevant 3D microenvironments.

\section{Larger scale techniques to elucidate mechanotransduction}

Of course, most cells reside within tissues, and the mechanical loads that they experience are often a consequence of macroscopic forces applied at the tissue-level. Many organs in the body perform physical functions (e.g., fluid transport, structural support, mechanical pumping), and early work in the field of biomechanics sought to understand how mechanical cues influence the behavior of physiological systems during homeostasis and disease [78]. Physical forces were shown to be indispensable to the maintenance and function of several organs in the body, and a variety of tissues - ranging from blood vessels to bone - were found to alter their structure in response to applied mechanical loads [78-80]

To investigate these effects experimentally, biomechanicians have used surgical techniques (e.g., blood vessel ligation) or in vivo mechanical testing (e.g., forelimb compression) 
to apply macroscopic forces to biological structures. Altered mechanical loads elicit an array of tissue-specific responses, such as changes in the expression of different signaling molecules, or modifications in the architecture of the tissue. Still, despite this progress, our understanding of mechanical feedback at the tissue-level remains largely phenomenological.

Recent experimental methods, which make use of developing embryos and sophisticated tissue culture techniques, offer exciting new potential to develop a more mechanistic understanding of tissue-level mechanotransduction. In these experimental systems, tissues consist primarily of epithelia. The relative architectural simplicity of these structures can be exploited to determine how mechanical forces are distributed within epithelial tissues, how groups of cells respond collectively to applied mechanical forces, and (ultimately) how these behaviors are coordinated to drive macroscopic changes in tissue form.

In general, these experimental methods come in two varieties (Fig. 4): those that measure the mechanical forces distributed within tissues, and those that use exogenous forces to control tissue behavior.

\section{Measuring mechanical stresses within epithelial tissues}

Within epithelia, macroscopic forces are distributed across the thickness of the tissue. Thus, the concept of mechanical stress, which describes a force vector acting over an area, is a useful normalized measure for mechanical loading. Mechanical stresses, however, cannot be measured directly, and must be inferred from deformations either within an epithelial tissue or within a substratum adherent to the tissue. 
Using developing embryos, several groups have used either micropipette punches [81, 82] or laser ablation [83-85] to induce wounds in epithelial tissues. The resulting wound geometry, as well as the tissue deformations around the wound, are then used to estimate the state of mechanical stress (Fig. 4A). These techniques can be used to measure how distributions of mechanical stress vary spatially across an epithelium or to determine how the state of stress at a particular point within the tissue changes over time.

Alternatively, distributions of mechanical stress within epithelial tissues can be computed by extending TFM to a multicellular context [86]. Recently, Tambe and colleagues used the motion of fluorescent beads embedded within a flexible substratum to compute the traction forces exerted by a collectively migrating epithelium [87]. In 2D culture, traction forces act along the basal surface of the epithelium. Thus, the computed forces were incorporated into a force balance for the epithelial layer and used to determine the distributions of mechanical stresses acting within the tissue (Fig. 4B). This new technique was termed monolayer stress microscopy, and the directed motion of collectively migrating cells within the epithelium was found to coincide with directions of principal stress [87].

In addition, our group recently extended TFM to the 3D culture of engineered epithelial tissues (Fig. 4C) [88-90]. Briefly, elastomeric stamps were used to create wells of defined shape in $3 \mathrm{D}$ collagen gels. Epithelial cells, which were seeded into the wells, then coalesced to form intact tissues. Fluorescent microspheres embedded within the surrounding collagen gel were used to estimate the mechanical forces exerted by both quiescent [89] and collectively migrating 
epithelial tissues [91] during 3D culture. This technique has been used to correlate levels of mechanical stress with changes in epithelial gene expression [92] and signal transduction [93].

Applying ectopic mechanical forces within epithelial tissues

In addition to measuring mechanical stresses within epithelial tissues, several recent studies have applied exogenous mechanical loads in an attempt to control the behavior and morphogenesis of embryonic tissues. For instance, Farge and colleagues used optical tweezers and microindentation to apply localized mechanical forces to developing epithelia in early Drosophila embryos [94-96]. These physical cues were shown to control patterns of epithelial gene expression [94], as well as the changes in cell shape that drive epithelial folding [95]. Brunet and colleagues extended this work to show that applied mechanical strains regulate mechanotransductive signaling pathways upstream of $\beta$-catenin in both early Drosophila and zebrafish embryos [96]. In addition, Kim and colleagues have developed a microfluidic device that flows a solution of ATP across a defined population of cells within a developing epithelium to locally induce cell contractility, and thereby alter patterns of mechanical stress within the tissue [97] (Fig. 4D). The mechanical stresses induced by these experimental techniques are not yet understood quantitatively, but could be combined with either laser ablation or TFM to develop a more complete understanding of mechanotransduction at the tissue level.

\section{Conclusion and future outlook}

Mechanotransduction, important in many biological contexts, occurs over a range of length scales. For this reason, experimental techniques are necessary to understand how mechanical stimuli arise on the scale of tissues, propagate across and within cells, and change 
molecular activity. This review sought to highlight many such techniques. Starting with the molecular scale, we highlighted recent studies using optical tweezers, magnetic tweezers, and FRET-based sensors. We then discussed techniques aimed at uncovering mechanotransduction on the level of individual cells, specifically TFM, deformable oil droplets, substrata with varying mechanical properties, micropipette aspiration, FRET-based sensors, and optogenetics. Finally, on scale of tissues, we highlighted methods involving multicellular TFM, microindentation, and microfluidic tissue culture.

Crossing multiple length scales in future experiments will help generate a better understanding of the interplay between biochemical and biophysical signaling, while potentially avoiding variation between experimental setups. Such experiments may include combining FRET-based sensors with monolayer stress microscopy, functionalized oil droplets with tissuelevel mechanical testing, and so on. In this light, work has combined FRET-based sensors with magnetic tweezers to measure changes in Cajal body protein activity in response to loads applied on the scale of the cell [98]. These multi-scale measurements and manipulations can then be integrated within hierarchical models of mechanotransduction [99]. In this way, by using the techniques described here either individually or in combination, we are well-poised to push, pull, and squeeze our way towards understanding mechanotransduction in many different biological contexts. 


\section{Acknowledgements}

Work from the authors' group was supported in part by grants from the NIH (GM083997,

HL110335, HL118532, HL120142), the NSF (CMMI-1435853), the David \& Lucile Packard

Foundation, the Alfred P. Sloan Foundation, and the Camille \& Henry Dreyfus Foundation.

C.M.N. holds a Career Award at the Scientific Interface from the Burroughs Wellcome Fund.

M.J.S. was supported in part by the NSF Graduate Research Fellowship Program. 


\section{References}

[1] T. Iskratsch, H. Wolfenson, M.P. Sheetz, Nat. Rev. Mol. Cell Biol., 15 (2014) 825-833.

[2] B.D. Hoffman, C. Grashoff, M.A. Schwartz, Nature, 475 (2011) 316-323.

[3] C.S. Chen, J. Tan, J. Tien, Annu. Rev. Biomed. Eng., 6 (2004) 275-302.

[4] C.J. Miller, L.A. Davidson, Nat. Rev. Genet., 14 (2013) 733-744.

[5] C. Bonnans, J. Chou, Z. Werb, Nat. Rev. Mol. Cell Biol., 15 (2014) 786-801.

[6] J. Seong, N. Wang, Y. Wang, J. Cell. Mol. Med., 17 (2013) 597-604.

[7] A.K. Simi, A.S. Piotrowski, C.M. Nelson, in: C. Maxwell, C. Roskelley (Eds.) Genomic Instability and Cancer Metastasis, Springer International Publishing2015, pp. 139-158.

[8] G. Charras, E. Sahai, Nat. Rev. Mol. Cell Biol., 15 (2014) 813-824.

[9] J.D. Humphrey, E.R. Dufresne, M.A. Schwartz, Nat. Rev. Mol. Cell Biol., 15 (2014) 802812.

[10] P. Tomakidi, S. Schulz, S. Proksch, W. Weber, T. Steinberg, Cell Tissue Res., 357 (2014) 515-526.

[11] B.C. Low, C.Q. Pan, G.V. Shivashankar, A. Bershadsky, M. Sudol, M. Sheetz, FEBS Lett., 588 (2014) 2663-2670.

[12] Y. Sun, K.M.A. Yong, L.G. Villa-Diaz, X. Zhang, W. Chen, R. Philson, S. Weng, H. Xu, P.H. Krebsbach, J. Fu, Nat. Mater., 13 (2014) 599-604.

[13] E.W. Gomez, Q.K. Chen, N. Gjorevski, C.M. Nelson, J. Cell. Biochem., 110 (2010) 44-51.

[14] E.N. Olson, A. Nordheim, Nat. Rev. Mol. Cell Biol., 11 (2010) 353-365.

[15] J. Li, B. Hou, S. Tumova, K. Muraki, A. Bruns, M.J. Ludlow, A. Sedo, A.J. Hyman, L. McKeown, R.S. Young, N.Y. Yuldasheva, Y. Majeed, L.A. Wilson, B. Rode, M.A. Bailey, H.R. Kim, Z. Fu, D.A.L. Carter, J. Bilton, H. Imrie, P. Ajuh, T.N. Dear, R.M. Cubbon, M.T. Kearney, K.R. Prasad, P.C. Evans, J.F.X. Ainscough, D.J. Beech, Nature, 515 (2014) 279-282.

[16] S.S. Ranade, S.-H. Woo, A.E. Dubin, R.A. Moshourab, C. Wetzel, M. Petrus, J. Mathur, V. Begay, B. Coste, J. Mainquist, A.J. Wilson, A.G. Francisco, K. Reddy, Z. Qiu, J.N. Wood, G.R. Lewin, A. Patapoutian, Nature, 516 (2014) 121-125.

[17] N.R. Blumenthal, O. Hermanson, B. Heimrich, V.P. Shastri, Proc. Natl. Acad. Sci. U. S. A., 111 (2014) 16124-16129.

[18] B.L. Pruitt, A.R. Dunn, W.I. Weis, W.J. Nelson, PLoS Biol., 12 (2014) e1001996. 
[19] M.A. Wozniak, C.S. Chen, Nat. Rev. Mol. Cell Biol., 10 (2009) 34-43.

[20] J.T. Parsons, A.R. Horwitz, M.A. Schwartz, Nat. Rev. Mol. Cell Biol., 11 (2010) 633-643.

[21] E.C. Yusko, C.L. Asbury, Mol. Biol. Cell, 25 (2014) 3717-3725.

[22] A. del Rio, R. Perez-Jimenez, R. Liu, P. Roca-Cusachs, J.M. Fernandez, M.P. Sheetz, Science, 323 (2009) 638-641.

[23] M.L. Smith, D. Gourdon, W.C. Little, K.E. Kubow, R.A. Eguiluz, S. Luna-Morris, V. Vogel, PLoS Biol., 5 (2007) e268.

[24] G. Baneyx, L. Baugh, V. Vogel, Proc. Natl. Acad. Sci. U. S. A., 99 (2002) 5139-5143.

[25] S. Sato, H. Inaba, Opt. Quant. Electron., 28 (1996) 1-16.

[26] D. Kilinc, G.U. Lee, Integr. Biol. (Camb.), 6 (2014) 27-34.

[27] K.C. Neuman, A. Nagy, Nat. Methods, 5 (2008) 491-505.

[28] C.D. Buckley, J. Tan, K.L. Anderson, D. Hanein, N. Volkmann, W.I. Weis, W.J. Nelson, A.R. Dunn, Science, 346 (2014) 1254211.

[29] W. Thomas, Annu. Rev. Biomed. Eng., 10 (2008) 39-57.

[30] C.T. Lim, Methods, (2015) Current Issue.

[31] R. Marjoram, Methods, (2015) Current Issue.

[32] Y. Wang, N. Wang, Integr. Biol. (Camb.), 1 (2009) 565-573.

[33] N. de Souza, Nat. Methods, 9 (2012) 873-877.

[34] A.-L. Cost, P. Ringer, A. Chrostek-Grashoff, C. Grashoff, Cell. Mol. Bioeng., 8 (2015) 96105.

[35] C. Berney, G. Danuser, Biophys. J., 84 (2003) 3992-4010.

[36] C. Grashoff, B.D. Hoffman, M.D. Brenner, R. Zhou, M. Parsons, M.T. Yang, M.A. McLean, S.G. Sligar, C.S. Chen, T. Ha, M.A. Schwartz, Nature, 466 (2010) 263-266.

[37] N. Borghi, M. Sorokina, O.G. Shcherbakova, W.I. Weis, B.L. Pruitt, W.J. Nelson, A.R. Dunn, Proc. Natl. Acad. Sci. U. S. A., 109 (2012) 12568-12573.

[38] M. Krieg, A.R. Dunn, M.B. Goodman, Nat. Cell Biol., 16 (2014) 224-233.

[39] T.J. Kim, S. Zheng, J. Sun, I. Muhamed, J. Wu, L. Lei, X.Y. Kong, D.E. Leckband, Y.X. Wang, Curr. Biol., 25 (2015) 218-224. 
[40] H. Chen, D.M. Cohen, D.M. Choudhury, N. Kioka, S.W. Craig, J. Cell Biol., 169 (2005) 459-470.

[41] E. Klotzsch, M.L. Smith, K.E. Kubow, S. Muntwyler, W.C. Little, F. Beyeler, D. Gourdon, B.J. Nelson, V. Vogel, Proc. Natl. Acad. Sci. U. S. A., 106 (2009) 18267-18272.

[42] G. Baneyx, L. Baugh, V. Vogel, Proc. Natl. Acad. Sci. U. S. A., 98 (2001) 14464-14468.

[43] V.D. Varner, C.M. Nelson, Integr. Biol. (Camb.), 5 (2013) 1162-1173.

[44] F. Meng, F. Sachs, J. Cell Sci., 125 (2012) 743-750.

[45] J. Guo, Y. Wang, F. Sachs, F. Meng, Proc. Natl. Acad. Sci. U. S. A., 111 (2014) E5252E5261.

[46] R.W. Style, R. Boltyanskiy, G.K. German, C. Hyland, C.W. MacMinn, A.F. Mertz, L.A. Wilen, Y. Xu, E.R. Dufresne, Soft Matter, 10 (2014) 4047-4055.

[47] W.R. Legant, C.K. Choi, J.S. Miller, L. Shao, L. Gao, E. Betzig, C.S. Chen, Proc. Natl. Acad. Sci. U. S. A., 110 (2013) 881-886.

[48] W.R. Legant, J.S. Miller, B.L. Blakely, D.M. Cohen, G.M. Genin, C.S. Chen, Nat. Methods, 7 (2010) 969-971.

[49] M. Dembo, T. Oliver, A. Ishihara, K. Jacobson, Biophys. J., 70 (1996) 2008-2022.

[50] J.L. Tan, J. Tien, D.M. Pirone, D.S. Gray, K. Bhadriraju, C.S. Chen, Proc. Natl. Acad. Sci. U. S. A., 100 (2003) 1484-1489.

[51] J.R.D. Soiné, C.A. Brand, J. Stricker, P.W. Oakes, M.L. Gardel, U.S. Schwarz, PLoS Comput. Biol., 11 (2015) e1004076.

[52] J. Toyjanova, E. Bar-Kochba, C. López-Fagundo, J. Reichner, D. Hoffman-Kim, C. Franck, PLoS One, 9 (2014) e90976.

[53] M.L. Rodriguez, B.T. Graham, L.M. Pabon, S.J. Han, C.E. Murry, N.J. Sniadecki, J. Biomech. Eng., 136 (2014) 051005.

[54] N.J.e.a. Sniadecki, Methods, (2015) Current Issue \#14.

[55] Y. Zhang, C. Ge, C. Zhu, K. Salaita, Nat. Commun., 5 (2014).

[56] M. Morimatsu, A.H. Mekhdjian, A.S. Adhikari, A.R. Dunn, Nano Letters, 13 (2013) 39853989.

[57] D.R. Stabley, C. Jurchenko, S.S. Marshall, K.S. Salaita, Nat. Methods, 9 (2012) 64-67.

[58] O. Campas, T. Mammoto, S. Hasso, R.A. Sperling, D. O'Connell, A.G. Bischof, R. Maas, D.A. Weitz, L. Mahadevan, D.E. Ingber, Nat. Methods, 11 (2014) 183-189. 
[59] J. Seong, M. Ouyang, T. Kim, J. Sun, P.-C. Wen, S. Lu, Y. Zhuo, N.M. Llewellyn, D.D. Schlaepfer, J.-L. Guan, S. Chien, Y. Wang, Nat. Commun., 2 (2011) 406.

[60] J. Seong, A. Tajik, J. Sun, J.-L. Guan, M.J. Humphries, S.E. Craig, A. Shekaran, A.J. García, S. Lu, M.Z. Lin, N. Wang, Y. Wang, Proc. Natl. Acad. Sci. U. S. A., 110 (2013) $19372-$ 19377.

[61] Y.-C. Poh, S. Na, F. Chowdhury, M. Ouyang, Y. Wang, N. Wang, PLoS One, 4 (2009) e7886.

[62] S. Na, O. Collin, F. Chowdhury, B. Tay, M. Ouyang, Y. Wang, N. Wang, Proc. Natl. Acad. Sci. U. S. A., 105 (2008) 6626-6631.

[63] Y. Tan, A. Tajik, J. Chen, Q. Jia, F. Chowdhury, L. Wang, J. Chen, S. Zhang, Y. Hong, H. Yi, D.C. Wu, Y. Zhang, F. Wei, Y.-C. Poh, J. Seong, R. Singh, L.-J. Lin, S. Doğanay, Y. Li, H. Jia, T. Ha, Y. Wang, B. Huang, N. Wang, Nat. Commun., 5 (2014).

[64] D.E. Discher, P. Janmey, Y.-1. Wang, Science, 310 (2005) 1139-1143.

[65] K. Lee, Q.K. Chen, C. Lui, M.A. Cichon, D.C. Radisky, C.M. Nelson, Mol. Biol. Cell, 23 (2012) 4097-4108.

[66] R.S. Stowers, S.C. Allen, L.J. Suggs, Proc. Natl. Acad. Sci. U. S. A., 112 (2015) 1953-1958.

[67] R.M. Hochmuth, J. Biomech., 33 (2000) 15-22.

[68] L.M. Lee, A.P. Liu, Lab Chip, 15 (2015) 264-273.

[69] A. Kumar, M. Mazzanti, M. Mistrik, M. Kosar, Galina V. Beznoussenko, Alexandre A. Mironov, M. Garrè, D. Parazzoli, G.V. Shivashankar, G. Scita, J. Bartek, M. Foiani, Cell, 158 (2014) 633-646.

[70] H. Tabdili, M. Langer, Q. Shi, Y.-C. Poh, N. Wang, D. Leckband, J. Cell Sci., 125 (2012) 4362-4371.

[71] A. Labernadie, A. Bouissou, P. Delobelle, S. Balor, R. Voituriez, A. Proag, I. Fourquaux, C. Thibault, C. Vieu, R. Poincloux, G.M. Charrière, I. Maridonneau-Parini, Nat. Commun., 5 (2014).

[72] R.e.a. Poincloux, Methods, (2015) Current Issue.

[73] L.J. Bugaj, A.T. Choksi, C.K. Mesuda, R.S. Kane, D.V. Schaffer, Nat. Methods, 10 (2013) 249-252.

[74] A.B. Jaffe, A. Hall, Annu. Rev. Cell Dev. Biol., 21 (2005) 247-269.

[75] A. Taslimi, J.D. Vrana, D. Chen, S. Borinskaya, B.J. Mayer, M.J. Kennedy, C.L. Tucker, Nat. Commun., 5 (2014). 
[76] E.S. Boyden, F. Zhang, E. Bamberg, G. Nagel, K. Deisseroth, Nat. Neurosci., 8 (2005) 1263-1268.

[77] K.A. Bell, H. Shim, C.-K. Chen, A.R. McQuiston, Neuropharmacology, 61 (2011) 13791388.

[78] Y.C. Fung, Biomechanics: motion, flow, stress, and growth, New York : SpringerVerlag1990.

[79] J.D. Humphrey, Cardiovascular solid mechanics : cells, tissues, and organs, 2002.

[80] S.C. Cowin, Annu. Rev. Biomed. Eng., 6 (2004) 77-107.

[81] V.D. Varner, L.A. Taber, Iutam Symposium on Cellular, Molecular and Tissue Mechanics, Proceedings, 16 (2010) 45-54.

[82] V.D. Varner, D.A. Voronov, L.A. Taber, Development, 137 (2010) 3801-3811.

[83] A.C. Martin, M. Gelbart, R. Fernandez-Gonzalez, M. Kaschube, E.F. Wieschaus, J. Cell Biol., 188 (2010) 735-749.

[84] M. Behrndt, G. Salbreux, P. Campinho, R. Hauschild, F. Oswald, J. Roensch, S.W. Grill, C.P. Heisenberg, Science, 338 (2012) 257-260.

[85] M. Smutny, M. Behrndt, P. Campinho, V. Ruprecht, C.-P. Heisenberg, in: C.M. Nelson (Ed.) Tissue Morphogenesis, Springer New York2015, pp. 219-235.

[86] X. Trepat, M.R. Wasserman, T.E. Angelini, E. Millet, D.A. Weitz, J.P. Butler, J.J. Fredberg, Nat. Phys., 5 (2009) 426-430.

[87] D.T. Tambe, C. Corey Hardin, T.E. Angelini, K. Rajendran, C.Y. Park, X. Serra-Picamal, E.H. Zhou, M.H. Zaman, J.P. Butler, D.A. Weitz, J.J. Fredberg, X. Trepat, Nat. Mater., 10 (2011) 469-475.

[88] N. Gjorevski, C.M. Nelson, Integr. Biol. (Camb), 2 (2010) 424-434.

[89] N. Gjorevski, C.M. Nelson, Biophys. J., 103 (2012) 152-162.

[90] A. Piotrowski, V. Varner, N. Gjorevski, C. Nelson, in: C.M. Nelson (Ed.) Tissue Morphogenesis, Springer New York2015, pp. 191-206.

[91] N.P. Gjorevski, A.; Varner, V.D.; Nelson, C.M., Sci. Rep., (2015) In press.

[92] K. Lee, N. Gjorevski, E. Boghaert, D.C. Radisky, C.M. Nelson, EMBO J., 30 (2011) 26622674.

[93] W. Zhu, C.M. Nelson, Dev. Biol., 379 (2013) 235-245. 
[94] N. Desprat, W. Supatto, P.A. Pouille, E. Beaurepaire, E. Farge, Dev. Cell, 15 (2008) 470477.

[95] P.A. Pouille, P. Ahmadi, A.C. Brunet, E. Farge, Sci. Signal., 2 (2009) ra16.

[96] T. Brunet, A. Bouclet, P. Ahmadi, D. Mitrossilis, B. Driquez, A.-C. Brunet, L. Henry, F. Serman, G. Béalle, C. Ménager, F. Dumas-Bouchiat, D. Givord, C. Yanicostas, D. Le-Roy, N.M. Dempsey, A. Plessis, E. Farge, Nat. Commun., 4 (2013) 2821.

[97] Y. Kim, M. Hazar, D.S. Vijayraghavan, J. Song, T.R. Jackson, S.D. Joshi, W.C. Messner, L.A. Davidson, P.R. LeDuc, Proc. Natl. Acad. Sci. U. S. A., 111 (2014) 14366-14371.

[98] Y.-C. Poh, S.P. Shevtsov, F. Chowdhury, D.C. Wu, S. Na, M. Dundr, N. Wang, Nat. Commun., 3 (2012) 866.

[99] J. Walpole, J.A. Papin, S.M. Peirce, Annu. Rev. Biomed. Eng., 15 (2013) 137-154. 


\section{Figure Legends}

\section{Figure 1: Mechanotransduction converts mechanical stimuli into instructions for}

biochemical signaling and gene expression. Proteins involved in mediating the cellular response to mechanical cues include stretch-activated ion channels, the transcription factors YAP/TAZ and MRTF-A, and FAK, a key component of cell-matrix adhesions.

Figure 2: Probing mechanotransduction on the molecular scale. (A) Optical tweezers use radiation pressure to exert force on a bead or particle. The schematic on the right depicts an experimental setup for investigating force-dependent formation of the cadherin-catenin complex. From [28]. Reprinted with permission from AAAS. (B) Magnetic tweezers apply force to superparamagnetic beads proportional to the gradient of a magnetic field. The schematic depicts an experimental setup for investigating force-dependent binding of vinculin to talin. From [22]. Reprinted with permission of AAAS. (C) FRET-based techniques provide a way to measure molecular scale forces and changes in conformation. Force can be calculated from FRET measurements by using a tension-sensing module inserted into a protein of interest (e.g. vinculin). Proper calibration provides a link between FRET efficiencies and forces. Adapted with permission from Macmillan Publishers Ltd: Nature [36], copyright 2010. Additionally, changes in fibronectin conformation have been observed by labeling the protein with a green donor and red acceptor fluorophore. Measurements of high FRET (red signal) near the cell interior suggest fibronectin exists in a compact state, whereas low FRET (green signal) near the cell periphery suggests the protein in that region is in an extended state. Adapted with permission [42]. Copyright by the National Academy of Sciences. 
Figure 3: Cellular scale techniques to investigate mechanotransduction. (A) Cell-level forces can be calculated by measuring displacements of embedded beads [46] or elastic micropillars [53]. These forces are a function of the displacements, elastic modulus $E$, and Poisson's ratio $v$. Displacements are exaggerated for ease of viewing. The scanning electron micrograph at right demonstrates the use of elastic micropillars to measure forces exerted by contracting smooth muscle cells. Adapted with permission [50]. Copyright by the National Academy of Sciences. (B) Stresses exerted by cells in vivo can be inferred by monitoring the deformations of fluorescent oil droplets conjugated with adhesion molecules. Adapted with permission from Macmillan Publishers Ltd: Nature Methods [58], copyright 2013. (C) Infrared radiation-mediated release of calcium or calcium chelators from liposomes provides spatiotemporal control of the moduli of alginate-based hydrogels [66]. (D) Micropipette aspiration was combined with microfluidics to apply suction pressure to an array of trapped cells. This technique was used to estimate the threshold cortical tension required to activate mechanosensitive ion channels, as seen by the uptake of a nuclear-localized, membrane-impermeable dye in the bottom image. Adapted from [68] with permission of The Royal Society of Chemistry. (E) Optogenetics provides a new way to precisely apply loads on the cellular scale. Shown here is a photoactivatable form of RhoA-mediated cellular contractility in which stress fiber formation is controlled by exposure to the photo-stimulus. Reprinted with permission from Macmillan Publishers Ltd: Nature Methods [73], copyright 2013.

Figure 4: Techniques to investigate mechanotransduction on the scale of tissues. (A) Laser microdissection used to probe the state of mechanical stress in the yolk syncytial layer (YSL) during early zebrafish development. (EVL; enveloping layer) Reprinted with permission from 
Springer Science and Business Media [85]. (B) Monolayer stress microscopy uses the traction forces obtained with TFM and incorporates them into a force balance for the epithelium to compute the 2D mechanical stress field within an epithelial layer. Reprinted with permission from Macmillan Publishers Ltd: Nature Materials [87], copyright 2011. (C) Extending TFM to 3D. The tracked motion of fluorescent microspheres embedded within the surrounding gel can be used to estimate the traction stresses induced by 3D micropatterned tissues in culture. With permission from Springer Science and Business Media [90]. (D) Microfluidic channels can be used to induce defined spatial patterns of contractility within developing epithelia. Reprinted with permission [39]. 
Figure 1

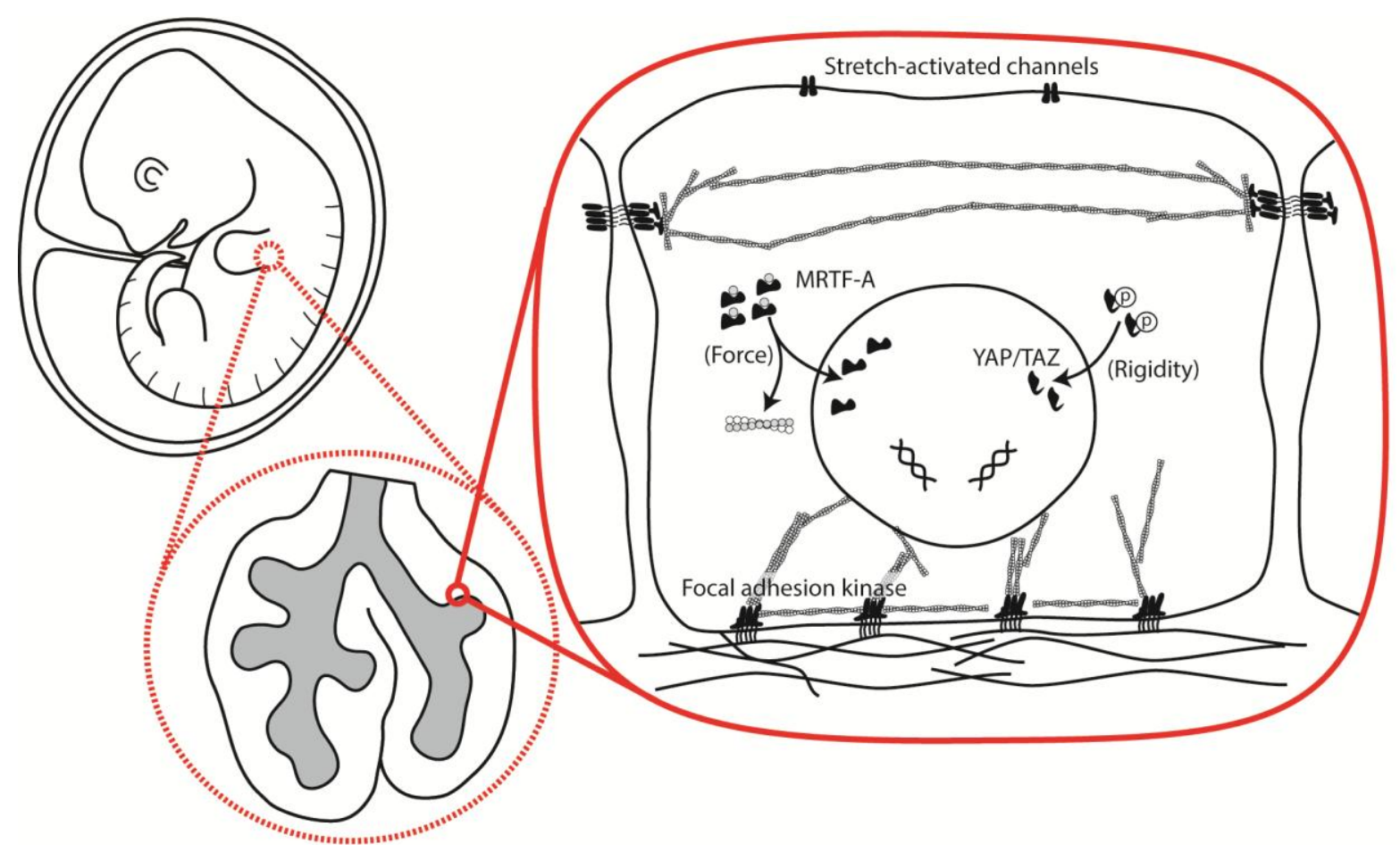




\section{Figure 2}
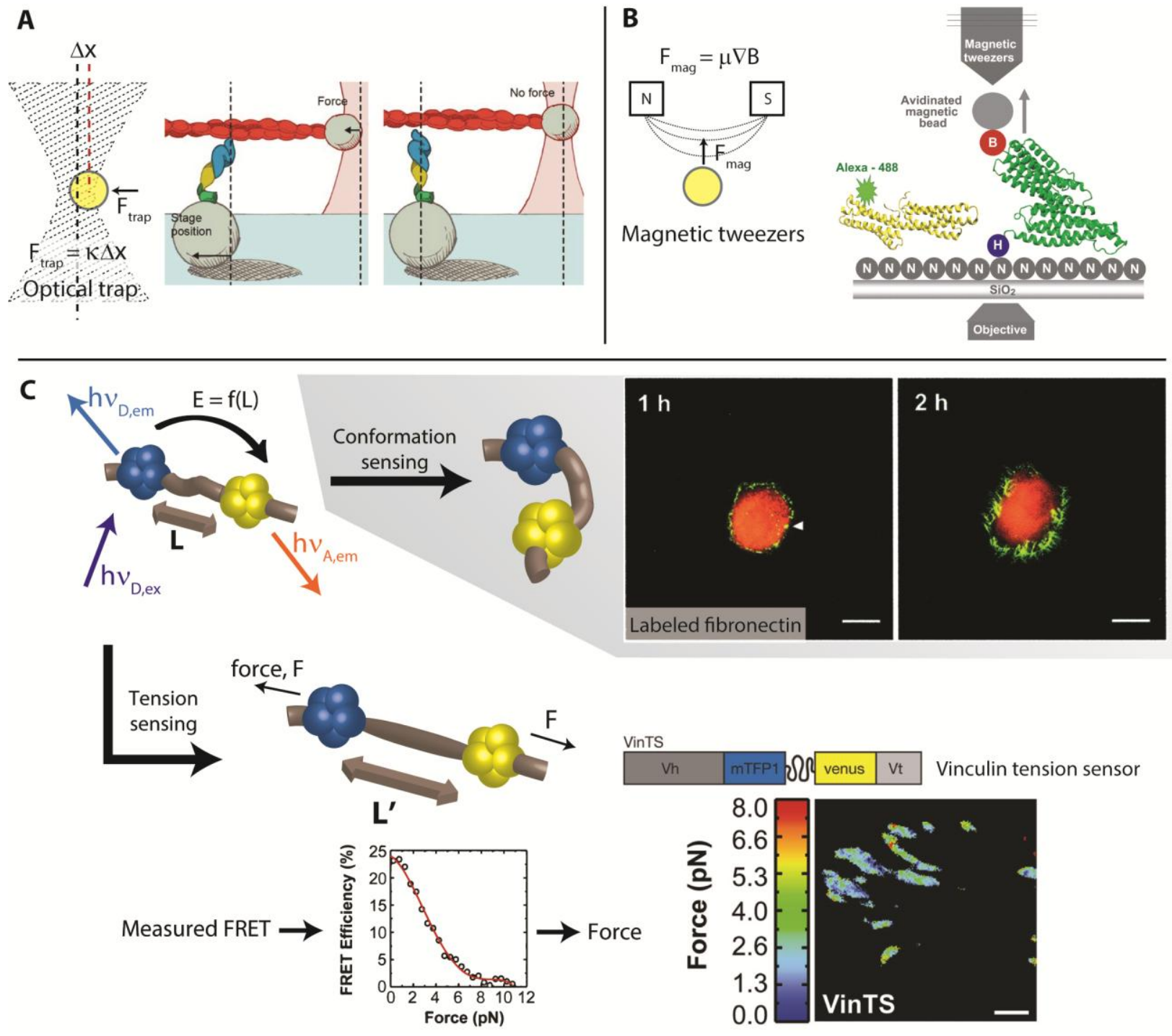


\section{Figure 3}

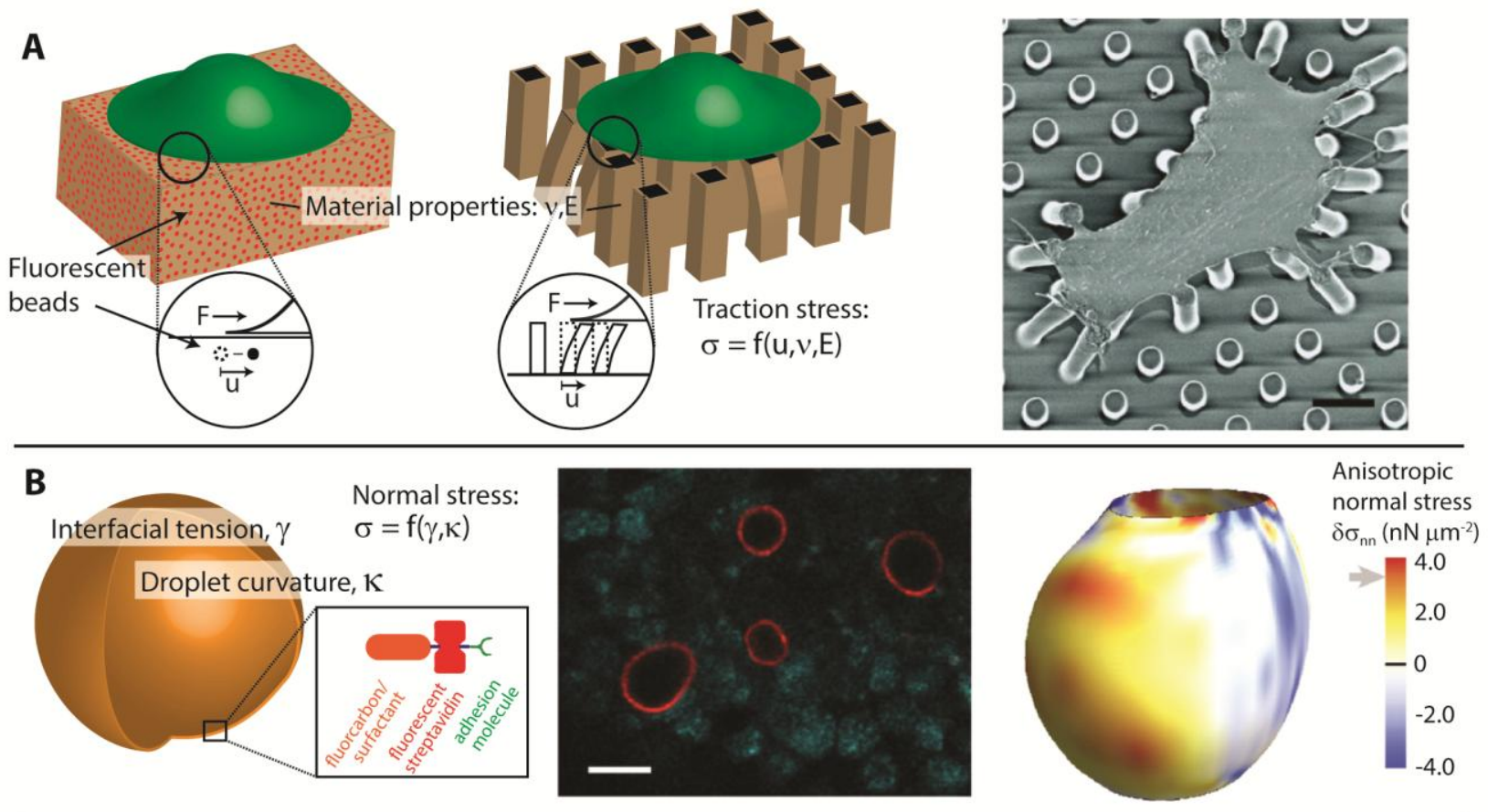

C
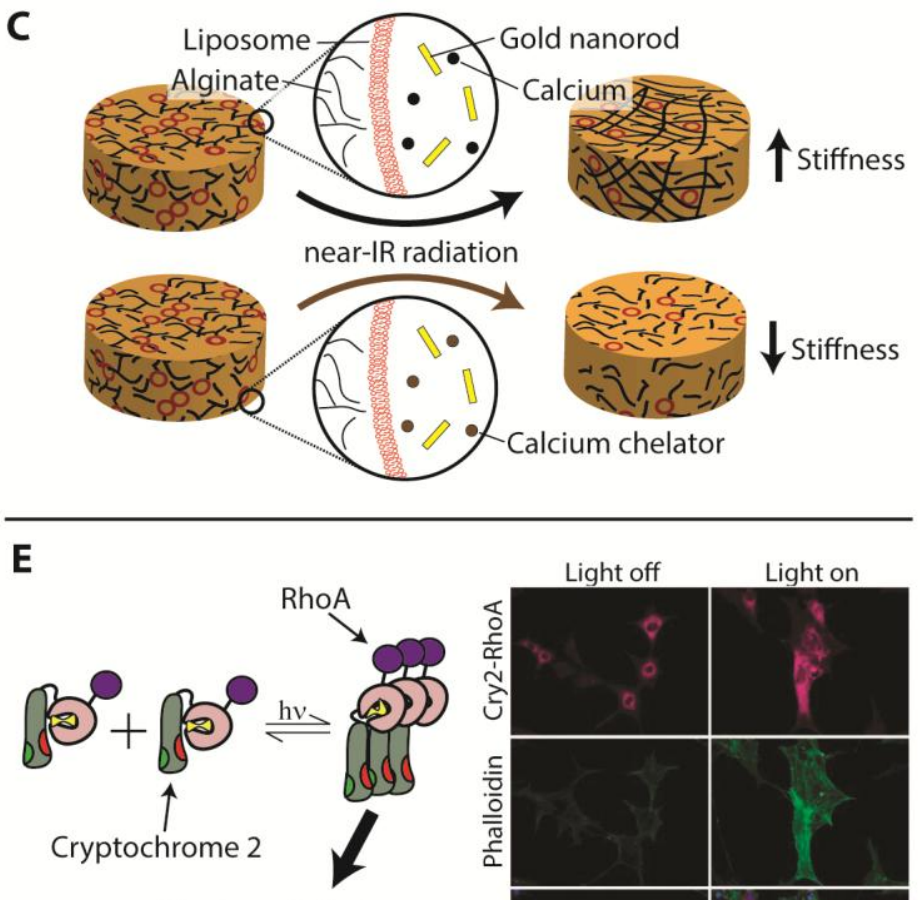

RhoA-mediated contractility and force generation

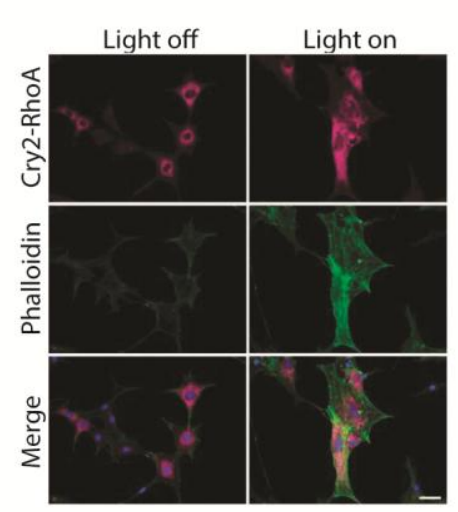


Figure 4
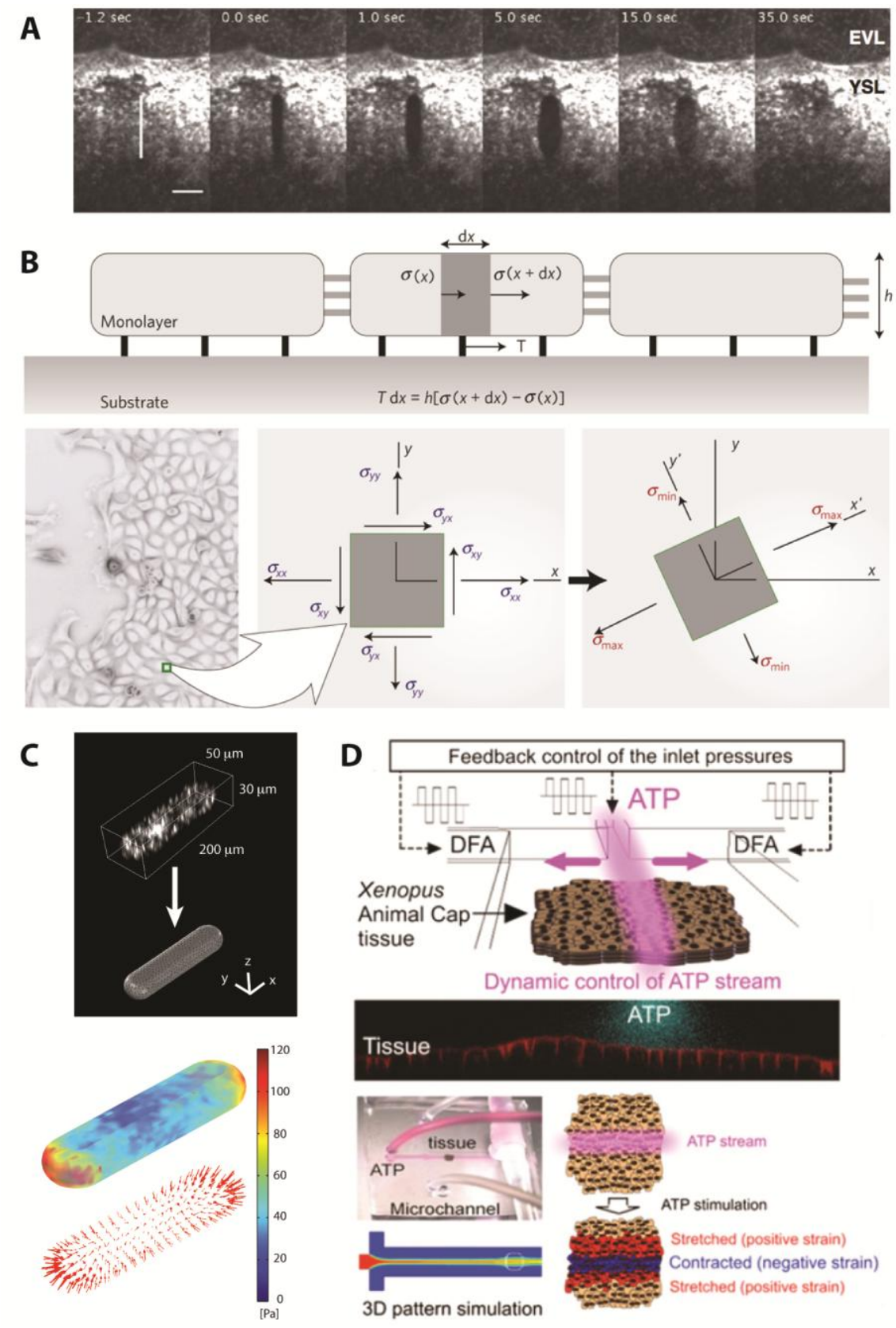